\title{
Transplante de células-tronco hematopoéticas - um estudo controlado sobre papéis ocupacionais ${ }^{1}$
}

\author{
Vivian Neves Dias ${ }^{a}$, Ana Paula Mastropietro ${ }^{\mathrm{b}}$, Érika Arantes de Oliveira Cardoso ${ }^{c}$, \\ Marysia Mara Rodrigues Prado De Carlo
}

aTerapeuta Ocupacional, Graduada, Faculdade de Medicina de Ribeirão Preto - FMRP, Universidade de São Paulo - USP, Ribeirão Preto, SP, Brasil

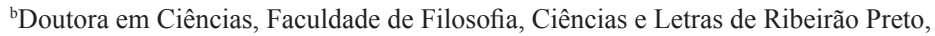
Terapeuta Ocupacional, Hospital das Clínicas, Faculdade de Medicina de Ribeirão Preto - FMRP, Universidade de São Paulo - USP, Ribeirão Preto, SP, Brasil

'Doutora em Psicologia, Psicóloga, Departamento de Psicologia e Educação, Faculdade de Filosofia,

Ciências e Letras de Ribeirão Preto, Universidade de São Paulo - USP, Ribeirão Preto, SP, Brasil dDoutora em Educação, Universidade Estadual de Campinas - UNICAMP, Pós-Doutora, Faculdade de Medicina, Universidade de São Paulo - USP, Docente, Divisão de Terapia Ocupacional, Departamento de Neurociências e

Ciências do Comportamento, Faculdade de Medicina de Ribeirão Preto - FMRP, Universidade de São Paulo - USP, Ribeirão Preto, SP, Brasil

\begin{abstract}
Resumo: O Transplante de Células-Tronco Hematopoiéticas é um procedimento que gera múltiplos sofrimentos ao paciente e seus familiares. Este estudo analisa as mudanças ocorridas nos papéis ocupacionais de pacientes submetidos ao transplante, na sua fase tardia (a partir de um ano pós-transplante), atendidos em um hospital universitário terciário, de grande porte, localizado no interior de São Paulo. Trata-se de um estudo quantitativo, baseado na aplicação de um protocolo internacional validado no Brasil - "Lista de Identificação de Papéis Ocupacionais". A casuística é composta por 52 sujeitos, sendo 26 do grupo de estudo e 26 do grupo-controle. Através da análise comparativa entre os grupos, constataram-se diferenças estatisticamente significativas entre os papéis ocupacionais de trabalhador, voluntário, amigo e participante em organizações. Concluiu-se que, passada a fase crítica pós-transplante, embora tenham ocorrido perdas, as pessoas transplantadas de medula óssea mudaram sua vida ocupacional, tendo resgatado ou mudado seus papéis ocupacionais.
\end{abstract}

Palavras-chave: Terapia Ocupacional, Transplante de Medula Óssea, Papel (Figurativo), Qualidade de Vida.

\section{Hematopoietic stem-cells transplants - a controlled study on the occupational roles}

\begin{abstract}
The transplant of hematopoietic stem-cells is a procedure that causes a lot of distress to the patient and family. This study analyses the changes occurred in the occupational roles of patients that have undergone transplants, in the late stage, assisted in a considerable university hospital located in the interior of the State of Sao Paulo. It is a quantitative research based on the use of an international protocol validated in Brazil: The Occupational Role Checklist. The survey consists of 52 subjects: 26 of the experimental group and 26 of the control group. Through the comparative analysis between the groups, statistically significant differences were observed between the occupational roles of workers, volunteers, friends and participants in organizations. It was possible to conclude that, after the critic stage right after transplant, although they have experienced some losses, the bone marrow transplanted subjects changed their occupational lives, having resumed or changed their occupational roles.
\end{abstract}

Keywords: Occupational Therapy, Bone Marrow Transplant, Occupational Roles, Quality of Life. 


\section{Introdução}

O Transplante de Células-Tronco Hematopoéticas, usualmente conhecido como transplante de medula óssea (TMO), é uma alternativa terapêutica eficaz quando os tratamentos convencionais não oferecem um bom prognóstico para vários tipos de neoplasias sólidas, hematológicas, doenças genéticas e imunológicas (OLIVEIRA-CARDOSO et al., 2009).

Os tipos de transplantes são: alogênico (as células-tronco hematopoiéticas provêm de um doador vivo, aparentado ou não aparentado, compatível, selecionado por testes de histocompatibilidade), autólogo (as células são retiradas do próprio paciente, são armazenadas e reinfundidas depois do regime de condicionamento), singênico (entre gêmeos univitelinos) e com células do cordáo umbilical (OKANE; MACHADO, 2009).

O transplante é dividido em três fases: Pré-Transplante Propriamente Dito e Pós-Transplante, dividido em Pós-Transplante Imediato (até cem dias depois do transplante) e Pós-Transplante Tardio, que é marcado pela alta do paciente do hospital-dia e o começo de seguimento no ambulatório (VOLTARELLI; STRACIERI, 2000; OLIVEIRA, 2004).

$\mathrm{Na}$ fase do pós-transplante tardia, a melhora gradual da condiçáo orgânica e do sistema autoimune possibilita a suspensão das drogas imunossupressoras, com a redução das restrições clínicas impostas pelo tratamento. Os pacientes transplantados, em geral, já retornaram às suas casas e procuram retomar suas atividades rotineiras, com a reestruturaçáo do cotidiano fora do hospital (MASTROPIETRO; SANTOS; OLIVEIRA, 2006).

\section{Objetivo}

Analisar as mudanças ocorridas nos papéis ocupacionais de pacientes submetidos ao transplante de células-tronco hematopoiéticas, na fase de pós-transplante tardio (a partir de um ano após o transplante).

\section{Procedimentos metodológicos}

Trata-se de um estudo quantitativo, cuja coleta de dados ocorreu através da aplicação de um protocolo internacional validado no Brasil - a "Lista de Identificação de Papéis Ocupacionais" (CORDEIRO et al., 2007). Foram avaliados dez papéis ocupacionais: Estudante, Trabalhador, Voluntário, Cuidador, Serviço doméstico, Amigo,
Membro familiar, Religioso, Passatempo/amador, Participante em Organizaçôes e "outro" (para descrever papéis que não estejam na lista). Foram também avaliadas a incumbência percebida e a importância designada aos papéis.

O estudo foi aprovado pelo Comitê de Ética em Pesquisa do hospital em questão (processo no 9109/2008) e recebeu fomento da Fundaçáo de Amparo à Pesquisa do Estado de São Paulo - FAPESP (bolsa de Iniciação Científica, processo no. 2009/50209-8). Todos os participantes foram esclarecidos e assinaram o Termo de Consentimento Livre e Esclarecido; após a assinatura, responderam todas as questóes do instrumento proposto.

Os sujeitos da pesquisa foram selecionados na fase pós-transplante tardia (pacientes com 1 ano ou mais após o transplante), através da agenda de retornos médicos do ambulatório da unidade. A coleta de dados ocorreu entre dezembro de 2008 e outubro de 2009.

No grupo de estudo, o protocolo foi aplicado em pacientes transplantados de células-tronco hematopoiéticas na fase tardia pós-transplante alogênico. No grupo controle, o protocolo foi aplicado em sujeitos não transplantados, que se dispuseram a participar da pesquisa, pareados ao grupo de estudo de acordo com idade, sexo e escolaridade.

De acordo com as características do protocolo utilizado, convencionou-se que a realização do Transplante é o marco na vida dos sujeitos do Grupo de Estudo, que separa o "passado" (antes da realização do transplante) do "presente" (os papéis que são/foram desempenhados depois do transplante até o dia em que a entrevista foi realizada) e o "futuro" e composto pelos papéis que o entrevistado pretende desempenhar a partir do dia seguinte da entrevista. Para o Grupo Controle, o marco de tempo é o período de um mês anterior à coleta de dados.

Os dados foram analisados estatisticamente pelo Teste Exato de Fisher, o Teste exato de Fisher-Freeman-Halton e o teste não paramétrico de Mann-Whitney.

\section{Resultados}

A casuística do estudo é composta por 52 sujeitos, sendo 26 do Grupo de Estudo (GE) e 26 do Grupo Controle (GC).

De acordo com os dados da Tabela 1 tanto o Grupo de Estudo como o Grupo Controle são compostos predominantemente por sujeitos casados e do sexo masculino, sendo que no primeiro a idade média é de 41,3 (variando entre 27 e 59 anos) e a escolaridade média de 8,3 anos e, no segundo grupo, 
a idade média é de 41,6 (variando entre 29 e 57 anos) e a escolaridade média de 9,8 anos. Por outro lado, no que se refere à ocupaçáo, enquanto no Grupo de Estudo apenas 15\% (4) são ativos, no Grupo Controle 96\% (25) são ativos.

Com a aplicação do protocolo "Lista de Identificação de Papéis Ocupacionais”, procurou-se identificar a média da distribuição desses papéis ao longo da vida, obtida através da soma do número de papéis desempenhados no passado, no presente e no futuro e, em seguida, dividido pelo número de sujeitos (26) de cada grupo. Os resultados obtidos, referentes ao grupo de estudo, foram: no passado, a média foi de 7,7 papéis desempenhados; no presente, foram 5,7; no futuro, foram 7,5. Em relação ao grupo controle, as médias de papéis ocupacionais foram: no passado, 8,2; no presente: 5,7 ; para o futuro: 7,7. Logo, observou-se que, em ambos os grupos, houve uma redução dos papéis do passado para o presente (desempenhavam mais papéis no passado do que no presente). Há expectativa de, no futuro, desempenhar mais papéis do que no presente, ainda que esta média seja menor que a de papéis desempenhados no passado.

Tabela 1. Características sociodemográficas do Grupo de estudo $(\mathrm{N}=26)$ e Grupo Controle $(\mathrm{N}=26)$.

\begin{tabular}{llcc}
\hline \multicolumn{2}{c}{ Variáveis } & GE & GC \\
\hline \multirow{2}{*}{ Idade } & Média & 41,3 & 41,6 \\
& Mediana & 40,0 & 39,5 \\
\hline \multirow{2}{*}{ Escolaridade } & Média & 8,3 & 9,8 \\
& Mediana & 7,5 & 10,5 \\
\hline \multirow{2}{*}{ Sexo } & Masculino & 15 & 15 \\
& Feminino & 11 & 11 \\
\hline \multirow{4}{*}{ Estado civil } & Solteiro & 4 & 6 \\
& Casado & 19 & 19 \\
& Separado & 0 & 1 \\
& Viúvo & 3 & 0 \\
\multirow{2}{*}{ Ocupação } & Ativo & 4 & 25 \\
& Inativo & 22 & 1 \\
\hline
\end{tabular}

Os resultados apresentados na Tabela 2, demonstram que há diferenças estatisticamente significativas entre o grupo de estudo e o grupo controle, relativas ao desempenho dos papéis ocupacionais de trabalhador (no tempo presente e futuro) e de voluntário (no tempo presente).

Houve diferença estatisticamente significativa $(p<0,001)$ no desempenho do papel de trabalhador no tempo presente dos sujeitos que realizam esse papel, sendo o percentual significantemente superior no grupo controle $96,2 \%$, enquanto que no grupo de estudo é de 30,8\%. O mesmo acontece no tempo futuro, em que $100 \%$ do grupo controle pretende desempenhar esse papel no futuro, no grupo de estudo apenas 69,2 pretendem desempenhá-lo, sendo o p-valor 0,004.

Em relação ao papel de voluntário, houve diferença estatisticamente significativa no tempo presente $(p=0,019)$, sendo o percentual significantemente superior no grupo de estudo que realiza esse papel $(38,5)$ em comparação ao grupo controle $(7,7)$.

$\mathrm{Na}$ Tabela 3, são apresentados os padróes de desempenho do papel de trabalhador e de voluntário.

Em relação ao papel de trabalhador, o padrão de desempenho C (contínuo) foi significativamente superior no grupo controle $(96,2 \%)$ em comparação com o do grupo de estudo (30,8\%). Já no padrão $\mathrm{P}$ (perda somente no presente) e PS (desempenhado somente no passado), houve uma porcentagem superior no grupo de estudo (respectivamente $38,5 \%$ e $26,9 \%$ ) em relação ao grupo controle (respectivamente $3,8 \%$ e $0 \%$ ).

Houve diferença estatisticamente significativa entre os grupos para o papel de voluntário $(p$-valor $=0,003)$, destacando-se o percentual do padrão $\mathrm{P}$ (perda somente no presente) foi maior no grupo controle $(42,3 \%)$ em comparação com o grupo de estudo $(3,8)$.

$\mathrm{Na}$ Tabela 4, são apresentados os dados referentes ao grau de importância designado a cada papel ocupacional.

Tabela 2. Comparação entre os grupos em relação ao papel de Trabalhador e Voluntário.

\begin{tabular}{|c|c|c|c|c|c|c|c|c|c|}
\hline & & & \multicolumn{2}{|c|}{ GE } & \multicolumn{2}{|c|}{ GC } & \multicolumn{2}{|c|}{ Total } & \multirow[b]{2}{*}{ P-valor } \\
\hline & & & $\mathbf{N}$ & $\%$ & $\mathbf{N}$ & $\%$ & $\mathbf{N}$ & $\%$ & \\
\hline \multirow{4}{*}{$\begin{array}{c}\text { Papel de } \\
\text { Trabalhador }\end{array}$} & \multirow{2}{*}{ Presente } & $0 *$ & 18 & 69,2 & 1 & 3,8 & 19 & 36,5 & \multirow{2}{*}{$<0,001$} \\
\hline & & $1 \dagger$ & 8 & 30,8 & 25 & 96,2 & 33 & 63,5 & \\
\hline & \multirow{2}{*}{ Futuro } & $0 *$ & 8 & 30,8 & 0 & 0 & 8 & 15,4 & \multirow{2}{*}{0,004} \\
\hline & & $1 \dagger$ & 18 & 69,2 & 26 & 100 & 44 & 84,6 & \\
\hline \multirow{2}{*}{$\begin{array}{c}\text { Papel de } \\
\text { Voluntário }\end{array}$} & \multirow{2}{*}{ Presente } & $0 *$ & 16 & 61,5 & 24 & 92,3 & 40 & 76,9 & \multirow{2}{*}{0,019} \\
\hline & & $1 \dagger$ & 10 & 38,5 & 2 & 7,7 & 12 & 23,1 & \\
\hline
\end{tabular}

*Não desempenha o papel ocupacional no tempo em questão. $†$ Desempenha o papel ocupacional no tempo em questão. 
Tabela 3. Comparação da distribuição do Padrão de Desempenho dos papéis ocupacionais de Trabalhador e de Voluntário.

\begin{tabular}{|c|c|c|c|c|c|c|c|c|}
\hline \multirow{3}{*}{$\begin{array}{c}\text { Papel } \\
\text { ocupacional }\end{array}$} & \multirow{3}{*}{$\begin{array}{l}\text { Padrão de } \\
\text { desempenho }\end{array}$} & \multicolumn{4}{|c|}{ Grupo } & \multirow{2}{*}{\multicolumn{2}{|c|}{ Total }} & \multirow[t]{3}{*}{ P-valor } \\
\hline & & \multicolumn{2}{|c|}{ GE } & \multicolumn{2}{|c|}{ GC } & & & \\
\hline & & $\mathbf{N}$ & $(\%)$ & $\mathbf{N}$ & $(\%)$ & $\mathbf{N}$ & $(\%)$ & \\
\hline \multirow{3}{*}{ Trabalhador } & $\mathrm{C} \ddagger$ & 8 & 30,8 & 25 & 96,2 & 33 & 63,5 & \multirow{3}{*}{0} \\
\hline & $\mathrm{P} \S$ & 10 & 38,5 & 1 & 3,8 & 11 & 21,2 & \\
\hline & PS\| & 7 & 26,9 & 0 & 0 & 7 & 13,5 & \\
\hline Voluntário & $P \S$ & 1 & 3,8 & 11 & 42,3 & 12 & 23,1 & 0,003 \\
\hline
\end{tabular}

†: Contínuo, §: Perda somente no presente, $\|$ : Desempenhado somente no passado.

Tabela 4. Grau de importância para os papéis de amigo e participante em organizações.

\begin{tabular}{lcccc}
\hline \multicolumn{1}{c}{ Grau de importância } & Grupos & Número de sujeitos & Total & P-valor \\
\hline \multirow{2}{*}{ Papel de amigo } & OII & 26 & 768,00 & \multirow{2}{*}{0,035} \\
& $1^{* *}$ & 26 & 610,00 & \\
\hline \multirow{2}{*}{ Papel de participante em organizações } & OII & 26 & 795,00 & \multirow{2}{*}{0,037} \\
\hline
\end{tabular}

II: o grupo de estudo e ${ }^{* *}$ : grupo controle.

De acordo com os dados da Tabela 4, os papéis de amigo e de participante em organizaçôes tiveram diferenças estatisticamente significativas - os valores do p-valor são respectivamente 0,035 e 0,037 . Os sujeitos do grupo de estudo obtiveram valores significativamente superiores ao grupo controle para ambos os papéis, ou seja, o grupo de estudo considera esses papéis mais importantes do que o grupo controle.

\section{Discussão}

De acordo com o "Modelo de Ocupação Humana" os papéis ocupacionais são os papéis que determinam a rotina diária e que organizam a maioria dos comportamentos da pessoa. As pessoas agem dentro de uma diversidade de papéis, que são experimentados todos os dias; não são apenas certas rotinas de ação habilidosa, mas envolvem também determinantes sobre quando elas são executadas, em qual contexto, com quem e qual a frequência (KIELHOFNER; BURKE, 1990).

A mudança nos papéis ocupacionais representa um processo adaptativo crítico, que pode ocorrer no contexto natural da ontogênese humana, como, por exemplo, a mudança do papel de estudante para o de trabalhador, mas que pode também ser consequência de condiçóes incapacitantes. A mudança de um papel para outro é um fenômeno complexo e requer a transformação dos hábitos e das habilidades para a integração de um novo padrão de vida diária (KIELHOFNER; BURKE, 1990).
O Transplante de Células-Tronco Hematopoiéticas é um procedimento complexo, utilizado como forma de tratamento para diversos tipos de patologias, malignas ou não, quando os tratamentos convencionais não têm um bom prognóstico. Este tipo de tratamento modifica significativamente o prognóstico de pacientes com doenças, como neoplasias e doenças hematológicas, que, até alguns anos atrás, eram fatais (SILVA, 2001). O câncer ou doenças hematológicas são doenças crônicas ainda muito associadas à dor, sofrimento e degradaçáo; receber um destes diagnósticos faz com que os pacientes se sintam diante da morte próxima, com sintomas aversivos, perda das habilidades funcionais, vocacionais, frustração e incertezas quanto ao futuro (MASTROPIETRO; OLIVEIRA; SANTOS, 2003).

Justamente por considerar a complexidade do transplante, é que é relevante avaliar o impacto do tratamento sobre o desempenho ocupacional desses sujeitos transplantados. Procurou-se verificar se os papéis ocupacionais tiveram mudanças e quais foram mais afetados, os que foram ganhos, perdidos e os que permaneceram contínuos e quais os graus de importância atribuídos a esses papéis.

Esse estudo teve como hipótese fundamental, confirmada, que o Transplante de Células-Tronco Hematopoéticas altera os papéis ocupacionais das pessoas a ele submetidas e que sua vida ocupacional continuará marcada por essa experiência, pois alguns dos papéis ocupacionais exercidos antes da realização do transplante não são retomados, mesmo num período tardio (após 1 ano de transplante). 
Através da apresentaçáo e análise dos dados quantitativos coletados, foram constatadas diferenças estatisticamente significativas em diversos papéis ocupacionais, seus padróes de desempenho e graus de importância. Os resultados deste estudo indicaram que houve uma perda de papéis ocupacionais, particularmente de trabalhador e estudante, em comparação com o tempo passado e que, para muitos, não houve a retomada de alguns papéis ocupacionais mesmo depois de alguns anos de transplante. Contudo, não ocorreram apenas perdas de papéis após o transplante; o estudo mostrou que o papel de voluntário é mais realizado no tempo presente pelos transplantados do que pelo grupo controle e também em comparação com o que era desempenhado antes do transplante.

Comparando as médias de papéis realizados no passado, no presente e no futuro, tanto do grupo de estudo como do grupo controle, verificou-se que houve perdas na passagem do passado para o presente e, em ambos os grupos, a média da expectativa para o futuro é a mesma. Uma das possibilidades para explicar esse resultado seria que as entrevistas foram realizadas com sujeitos que apresentaram uma variação muito grande no tempo após o transplante - de um ano e um mês até 14 anos após o transplante - sendo que, quanto mais longo o tempo transcorrido, mais parecidos se tornam os desempenhos de papéis ocupacionais dos sujeitos dos grupos: de estudo e controle.

Pode-se perceber, através deste estudo, que os sujeitos transplantados náo tiveram apenas perdas em seus papéis ocupacionais após o transplante; houve ganho de papéis, como os de voluntário, serviços domésticos, cuidador, religioso e participante em organizaçóes. Além disto, constatou-se que não são apenas os transplantados que sofreram perdas em seus papéis ocupacionais, mas também os sujeitos do grupo controle tiveram perdas.

O papel ocupacional que teve maior perda foi o de estudante e, além disso, ao avaliar o grau de importância atribuído, verificou-se que $92 \%$ dos sujeitos do grupo de estudo consideraram o papel de trabalhador como muito importante.

O trabalho tem papel central na constituição da identidade individual e pode favorecer as diversas formas de inserção social dos sujeitos, sendo muito importante na constituição de redes de relaçóes sociais e de trocas afetivas e econômicas, que sáo a base da vida cotidiana das pessoas. Num processo de busca de semelhanças e diferenças, a partir das trocas materiais e afetivas, o sujeito constitui sua singularidade (LANCMAN, 2007).
O processo produtivo está relacionado a aspectos fisiológicos, sociais e econômicos, como também com a afirmação da autoestima perante a sociedade. O trabalho é visto como o principal organizador da vida humana e ocupa um importante e significativo espaço na vida da maioria das pessoas, pois, na sociedade atual, a pessoa é vista conforme o papel que ocupa na organizaçáo, seu local e poder que desempenha. Ao se aposentar, grande número de pessoas perde seu ponto de referência (ROMANINI; XAVIER; KOVALESKI, 2004).

Em relação aos sujeitos deste estudo, alguns aposentaram e outros estáo afastados do trabalho. Portanto, pode-se inferir, em função do que foi discutido acima, que tenha sido muito difícil para eles a perda do papel de trabalhador em suas vidas, que eles consideram como muito importante.

Outros papéis que os sujeitos do grupo de estudo consideraram como muito importantes foram: de membro familiar, de amigo e de cuidador, contudo, esses papéis se mantiveram contínuos ao longo do tempo. Os sujeitos do grupo controle consideram como mais importantes os papéis de estudante, trabalhador e membro familiar, sendo que o papel de estudante não permaneceu contínuo, ao contrário dos outros dois que foram contínuos ao longo do tempo.

Pode-se observar, através da comparação entre os dois grupos, que os papéis que tiveram uma diferença significativa foram os papéis de trabalhador e o de voluntário para sua realização durante o tempo, e os papéis de amigo e participante em organizaçóes para a importância designada.

Em relação ao papel de trabalhador, a diferença entre os grupos foi no tempo presente e no tempo futuro, sendo maior no grupo controle a execução do papel e sua expectativa para o futuro. Sobre o padráo de desempenho do mesmo papel, o percentual para o padrão contínuo é superior no grupo controle do que no grupo de estudo. No grupo de estudo, a porcentagem do padrão de desempenho com perda somente no presente e desempenhado somente no passado é maior que no grupo controle.

O papel de voluntário no tempo presente é maior no grupo de estudo do que no grupo controle. No padrão de desempenho, a perda somente no presente foi superior no grupo controle quando comparado com o grupo de estudo. Os motivos que podem ter levado o grupo de estudo a realizar mais este papel poderiam estar relacionados ao fato de que as pessoas teriam mais tempo para realizar outras atividades e papéis que não poderiam fazer antes, trocando o trabalho assalariado por um trabalho voluntário, inclusive devido à aposentadoria e gratidão pelo transplante. 
Em relação ao grau de importância para cada papel ocupacional, o de amigo e participante em organizações teve uma importância maior para o grupo de estudo do que para o grupo controle, o que também pode estar relacionado ao fato de ter passado pelo transplante e por dar mais valor às pessoas, aos amigos, mais do que os sujeitos que não passaram por esta experiência. Outro motivo seria que eles têm mais tempo para fazer amizades, já que a maioria dos sujeitos entrevistados está afastada do trabalho ou aposentada.

Por fim, como referido na literatura, o comportamento ocupacional envolve a motivação, os hábitos e papéis, e as capacidades, que precisam trabalhar em harmonia entre si, possibilitando contribuiçôes simultâneas ao comportamento (BARRETT; KIELHOFNER, 2002). Portanto, as pessoas que vivenciaram o transplante enfrentaram várias mudanças no seu modo de viver, no desempenho de seus papéis ocupacionais e no valor a eles conferido.

\section{Considerações finais}

Através desse trabalho pode-se concluir que o Transplante de Células-Tronco Hematopoiéticas é um procedimento altamente complexo, que gera múltiplos sofrimentos ao transplantado e seus familiares. Porém, quando passada a fase crítica do tratamento, as pessoas transplantadas retomaram a maioria dos seus papéis ocupacionais, ainda que com algumas perdas, mas também com alguns ganhos de novos papéis.

Para atingir os objetivos dessa pesquisa dois aspectos metodológicos merecem ser destacados: a importância da realização do projeto-piloto, por possibilitar o contato com o protocolo e com as pessoas, para identificar previamente as principais dificuldades que o sujeito e o pesquisador teriam e para tirar as dúvidas da pesquisadora antes da coleta de dados propriamente dita.

Este trabalho foi desenvolvido numa área de conhecimentos ainda com escassa produção científica, sendo, portanto, necessários mais estudos científicos para o aprofundamento das investigaçóes sobre a vida e o desempenho ocupacional desta populaçáo. Neste sentido, o profissional terapeuta ocupacional é de extrema importância na equipe multiprofissional que atende os sujeitos transplantados, para a promoçáo de sua qualidade de vida.

A Terapia Ocupacional através das intervenções terapêuticas, utilizando as atividades humanas de um modo geral, desenvolve condiçóes e oportunidades para a autonomia e integração do sujeito, possibilitando uma melhor qualidade de vida (FERRER; SANTOS, 2007). É primordial a atuação do terapeuta ocupacional em programas de assistência integral à saúde pela promoção da vida ocupacional do sujeito em vários aspectos. Atuando tanto na prevenção, no tratamento como na aquisição de independência e autonomia do sujeito (DE CARLO; MIOSHI; NICOLAU, 2000).

A atuação da Terapia Ocupacional na área de oncologia tem como objetivo fazer com que o sujeito atinja suas capacidades funcionais, ocupacionais com autonomia e independência nas atividades de sua vida. Pode acontecer em todas as fases do tratamento, inclusive quando os sujeitos estão em cuidados paliativos. A atuaçấo da terapia ocupacional com sujeitos que realizaram o transplante de células-tronco hematopoiéticas no contexto hospitalar pode atuar na internação, no pré-transplante, no pós-transplante e na programação de alta (PALM, 2007).

Os sujeitos que vivenciam o transplante de células-tronco hematopoiéticas têm uma grande necessidade de reconstruir seu cotidiano. Passar por uma doença grave que muitas vezes é fatal e seguir um tratamento invasivo modifica a vida, a identidade pessoal e profissional, provoca uma ruptura no cotidiano, porém possibilita também construir um novo cotidiano muitas vezes com a finalidade de superar as dificuldades da reinserção social (MASTROPIETRO, 2009).

De acordo com Bennett et al. (1990 apud IMAJÓ, 2007), na fase de pós transplante de células-tronco hematopoiéticas tardia, o terapeuta ocupacional tem como objetivo de sua atuação a independência do sujeito em diversas atividades, sejam elas as atividades de vida diária, lazer, trabalho, estudo. Dentre os muitos procedimentos a serem realizados, o terapeuta ocupacional pode orientar o sujeito na prevenção de contraturas em decorrência a esclerodermias e, quando preciso, confeccionar órteses, fazer adaptaçôes e mesmo favorecer o encaminhamento e continuidade do tratamento em outros serviços da comunidade.

Portanto, o terapeuta ocupacional pode estar presente em todo o processo de reconstrução da vida ocupacional do sujeito, ajudando-o a estabelecer suas metas terapêuticas e, de uma forma gradual, levando-o a considerar suas limitaçóes. $\mathrm{O}$ terapeuta ocupacional o ajuda a refletir sobre sua experiência de vida relacionada ao transplante, para que seja autor de sua própria vida (MASTROPIETRO; OLIVEIRA; SANTOS, 2003). 


\section{Referências}

BARRETT, L.; KIELHOFNER, G. Teorias Derivadas de Perspectivas do Comportamento Ocupacional. In: NEISTADT, M. E.; CREPEAU, E. B. (Orgs.). Williard \& Spackman - Terapia Ocupacional. Rio de Janeiro: Guanabara Koogam; 2002. p. 488-497.

CORDEIRO, J. J. R. et al. Cross-Cultural Reproducibility of the Brazilian Portuguese Version of the Role Checklist for Persons With Chronic Obstructive Pulmonary Disease. American Journal of Occupational Therapy, New York, v. 61, n. 1, p. 33-40, 2007. PMid:17302103. http:// dx.doi.org/10.5014/ajot.61.1.33

DE CARLO, M. M. R. P.; MIOSHI, E.; NICOLAU, S. N. Projeto de Implantaçáo do Programa Didático-assistencial de Terapia Ocupacional no Hospital Universitário da Universidade de São Paulo. São Paulo: EdUSP, 2000. 27 p.

FERRER, A. L.; SANTOS, W. A. Terapia Ocupacional na Atenção a Pacientes com Dor Oncológica e em Cuidados Paliativos. In: DE CARLO, M. M. R. P.; DE QUEIROZ, M. E. G. (Orgs.). Dor e Cuidados Paliativos: Terapia Ocupacional e Interdisciplinaridade. São Paulo: Roca, 2007. p. 146-166.

IMAJÓ, J. A inserção da Terapia Ocupacional em Centros de Transplante de Medula Óssea brasileiros. 2007. 59 f. Monografia (Graduação em Terapia Ocupacional)Faculdade de Medicina de Ribeirão Preto, Universidade de São Paulo, Ribeirão Preto, 2007.

KIELHOFNER, G.; BURKE, J. Modelo da ocupação humana: parte I. Revista de Terapia Ocupacional da Universidade de São Paulo, São Paulo, v. 1, n. 1, p. 55-67, 1990.

LANCMAN, S. Psicodinâmica do Trabalho. In: CAVALCANTI, A.; GALVÃO, C. Terapia Ocupacional: fundamentação \& prática. Rio de Janeiro: Guanabara Koogan, 2007. p. 271-277.

MASTROPIETRO, A. P. Ações da Terapia Ocupacional na Unidade de Transplante de Medula Óssea (UTMO) do HCFMRP-USP. In: FIGUEIREDO, L. R. U.; NEGRINI, S. F. B. M. (Orgs.). Terapia Ocupacional: Diferentes práticas em hospital geral. Ribeirão Preto: Legis Summa, 2009. p. 211-223.
MASTROPIETRO, A. P.; OLIVEIRA, E. A.; SANTOS, M. A. Reinserção profissional de pacientes submetidos ao transplante de medula óssea. Revista Centro Universitário Claretiano Batatais, Batatais, n. 3, p. 139-45, jan. 2003.

MASTROPIETRO, A. P.; SANTOS, M. A.; OLIVEIRA, E. A. Sobreviventes do transplante de medula óssea: construção do cotidiano. Revista de Terapia Ocupacional da Universidade de São Paulo, São Paulo, v. 17, n. 2, p. 64-71, 2006.

OKANE, E. S. H.; MACHADO, L. N. Histórico. In: MACHADO, L. N. et al. (Orgs.). Transplante de medula óssea: abordagem multidisciplinar. São Paulo: Lemar, 2009. p. 23-29.

OLIVEIRA, E. A. Qualidade de vida de paciente submetidos ao transplante de medula óssea alogênico: um estudo longitudinal. 2004. 276 f. Tese (Doutorado em Psicologia)Faculdade de Filosofia, Ciências e Letras de Ribeirão Preto, Universidade de São Paulo, Ribeiráo Preto, 2004.

OLIVEIRA-CARDOSO, E. A. et al. Qualidade de vida de sobreviventes do transplante de medula óssea (TMO): um estudo prospectivo. Psicologia: Teoria e Pesquisa, v. 25, n. 4, p. 621-628, 2009. Disponível em: <http://www.scielo.br/scielo.php?script=sci_arttext\&p $\mathrm{id}=$ S0102-37722009000400018 >. Acesso em: 20 out. 2010.

PALM, R. D. C. M. Oncologia. In: CAVALCANTI, A.; GALVÃO, C. Terapia Ocupacional: fundamentação \& prática. Rio de Janeiro: Guanabara Koogan, 2007. p. 487-492.

ROMANINI, D. P.; XAVIER, A. A. P.; KOVALESKI, J. L. Aposentadoria: período de transformações e preparação. In: ENCONTRO NACIONAL DE ENGENHARIA DE PRODUÇÃO - ENEGEP, 24., 2004, Florianópolis. Anais... Florianópolis: UFSC; ABEPRO, 2004.

SILVA, L. M. G. Breve reflexão sobre autocuidado no planejamento de alta hospitalar pós-transplante de medula óssea (TMO): relato de caso. Revista latino-americana de Enfermagem, Ribeirão Preto, v. 9, n. 4, p. 75-82, 2001.

VOLTARELLI, J. C.; STRACIERI, A. B. P. L. Aspectos imunológicos dos transplantes de células tronco hematopoiéticas. Medicina, v. 33, n. 4, p. 443-462, 2000.

\section{Contribuição dos Autores}

V. N. Dias participou da coleta, elaboração e análise dos dados e redação do artigo; A. P. Mastropietro e É. A. O. Cardoso colaboraram na seleçấo dos sujeitos da pesquisa e na coleta de dados; M. M. R. P. De Carlo, como orientadora do trabalho, colaborou no desenho do estudo, na análise dos dados e correção do manuscrito e na revisão crítica do artigo.

\section{Notas}

${ }^{1}$ Baseado na pesquisa de Iniciação Científica, financiado pela Fundação de Amparo à Pesquisa do Estado de São Paulo - FAPESP, processo $n^{\circ}$. 2009/50209-8. 